J. Indones. Math. Soc.

Vol. 23, No. 2 (2017), pp. 33-45.

\title{
ON FULLY PRIME RADICALS
}

\author{
IndAh EMILIA WiJAYANTi ${ }^{1}$ AND Dian ARIEsta YuWANingsiH ${ }^{2}$ \\ ${ }^{1,2}$ Department of Mathematics, \\ Universitas Gadjah Mada, Yogyakarta, Indonesia \\ ${ }^{1}$ ind_wijayanti@ugm.ac.id \\ ${ }^{2}$ dian.ariesta17@yahoo.com
}

\begin{abstract}
In this paper we give a further study on fully prime submodules. For any fully prime submodules we define a product called $*_{M}$-product. The further investigation of fully prime submodules in this work, i.e. the fully m-system and fully prime radicals, is related to this product. We show that the fully prime radical of any submodules can be characterized by the fully m-system. As a special case, the fully prime radical of a module $M$ is the intersection of all minimal fully prime submodules of $M$.

Key words and Phrases: Fully invariant submodules, fully prime submodules, fully $\mathrm{m}$-system, fully prime radicals.
\end{abstract}

\begin{abstract}
Abstrak. Pada tulisan ini disajikan hasil penelitian terkait submodul prima penuh. Didefinisikan operasi perkalian- $*_{M}$ untuk setiap submodul prima penuh. Penelitian lebih lanjut terkait submodul prima penuh, yaitu sistem- $m$ penuh dan radikal prima penuh, dapat dihubungkan dengan operasi perkalian- $*_{M}$ ini. Lebih lanjut, dapat ditunjukkan bahwa radikal prima penuh dari sebarang submodul dapat dibentuk dari sistem- $m$ penuh. Lebih lanjut, diperoleh bahwa radikal prima penuh dari suatu modul $M$ merupakan irisan dari semua submodul prima penuh minimal di $M$.

Kata kunci: Submodul invarian penuh, submodul prima penuh, sistem- $m$ penuh, radikal prima penuh.
\end{abstract}

\section{INTRODUCTION}

Along with the process of generalization of rings to modules, some previous authors defined prime submodules as the generalization of prime ideals. By using some different approaches, there are some kinds of definition of prime submodules.

2000 Mathematics Subject Classification: 16D10.

Received: 14 Sept 2016, revised: 4 Sept 2017, accepted: 31 Oct 2017. 
The definition of prime submodules introduced by Dauns [2] has been referred by many authors for their study of primeness in module theory. Investigations of prime radicals and localization of modules by Wisbauer [11] are also based on this definition.

In this work we refer to the definition of primeness of submodules based on the paper of Wijayanti and Wisbauer [8], in which they defined prime (sub)module using the $*_{M}$-product and called it fully prime submodules. This $*_{M}$-product was introduced earlier by Raggi et al. in their paper [7] and recently gives a possibility in a module to have such a "multiplication", such that we can adopt the definition of prime ideal to the module theory easier. The further study of the fully prime submodules started in paper Wijayanti [9]. The aim of this work is to complete the investigation of fully prime submodules, especially related to the m-system and radical.

Some previous authors also studied the prime submodules and prime radical submodules, for example Sanh [6], Lam [4] and Azizi [1]. We refer to Lam for defining fully m-systems and Sanh [6] for some properties of fully prime radicals of submodules.

In the next section we present some necessary and sufficient conditions of fully prime submodules in Proposition 2.2 and Proposition 2.5. Moreover, we show that there is a fully prime submodule which is not a prime submodule by giving a counter example in Example 2.3. To give some ideas of the fully primeness among another primeness, i.e. primeness in the sense of Dauns [2] and Sanh [5], we prove in Proposition 2.7, Proposition 2.9 and Lemma 2.10 that they not necessary coincide. Then we define a fully m-system and show that it is a complement of the fully prime submodule (see Proposition 2.13).

In the last section we define fully prime radicals and give some results. In Proposition 3.1 we characterize the fully prime radical of a submodule using the fully m-system. Moreover, in Proposition 3.9 we show that if the module is selfprojective, then the fully prime radical of factor module modulo its fully prime radical is equal to zero.

Throughout $R$ denotes an associative ring with unit and the module should be a unital left module over the related ring. For our purpose, we write the homomorphism on the right side. A fully invariant submodule $K$ in $M$ is a submodule which satisfies $(K) f \subseteq K$ for any endomorphism $f$ of $M$. Naturally, for any $R$-module $M$, it is also a right $S$-module, where $S=\operatorname{End}_{R}(M)$ and the scalar multiplication is defined as $\mu:(m, f) \mapsto(m) f$.

\section{Fully Prime Submodules}

We begin this section by the definition of a product between two fully invariant submodules as we refer to [7] and [8]. For any fully invariant submodules $K, L$ of $M$, consider the product

$$
K *_{M} L:=K \operatorname{Hom}_{R}(M, L) .
$$


We call the defined product in formulae (1) as the $*_{M}$-product. Now we recall the definition of fully prime submodules in [8].

Definition 2.1. A fully invariant submodule $P$ of $M$ is called a fully prime submodule in $M$ if for any fully invariant submodules $K, L$ of $M$,

$$
K *_{M} L \subseteq P \Rightarrow K \subseteq P \text { or } L \subseteq P .
$$

A module $M$ is called a fully prime module if its zero submodule is a fully prime submodule.

Moreover, we give a characterization of fully prime submodules as we can show in the following proposition.

Proposition 2.2. Let $P$ be a fully invariant submodule of $M$. The following assertions are equivalent:
a. $P$ is a fully prime submodule;
b. For any $m, k \in M$ and fully invariant cyclic submodules $\langle m\rangle$ and $<k>$ of $M$, if $<m>*_{M}<k>\subseteq P$, then $m \in P$ or $k \in P$.
c. For any submodules $K, L$ of $M$, if $K S *_{M} L S \subseteq P$, then $K \subseteq P$ or $L \subseteq P$.

Proof. (a) $\Rightarrow$ (b). Let $m$ and $k$ be elements in $M$ where the fully invariant cyclic submodules $<m>*_{M}<k>\subseteq P$. Since $P$ is fully prime, it implies $<m>=$ $m S \subseteq P$ or $\langle k\rangle=k S \subseteq P$. Then $m \in P$ or $k \in P$.

(b) $\Rightarrow$ (c). Let $K$ and $L$ be $R$-submodules of $M$ where $K S *_{M} L S \subseteq P$. Take any $k \in K$ and $l \in L$, then $k S \subseteq K S$ and $l S \subseteq L S$. Moreover we have

$$
k S *_{M} l S \subseteq K S *_{M} L S \subseteq P,
$$

and it implies $k \in P$ or $l \in P$. Thus $K \subseteq P$ or $L \subseteq P$.

(c) $\Rightarrow$ (a). Let $K, L$ be fully invariant submodules of $M$ where $K *_{M} L \subseteq P$. Since $K S \subseteq K$ and $L S \subseteq L$, then $K S *_{M} L S \subseteq K *_{M} L \subseteq P$. Thus we have $K \subseteq P$ or $L \subseteq P$.

Example 2.3. Let us give an example of a submodule which is not prime but fully prime. In $\mathbb{Z}$-module $\mathbb{Z}_{12}$, we know that $\langle\overline{3}\rangle$ is not a prime submodule. But $<\overline{3}>$ is a fully prime submodule, since for any proper submodules $N, K$ in $\mathbb{Z}_{12}$, if $N *_{\mathbb{Z}_{12}} K \subseteq<\overline{3}>$, then $N \subseteq<\overline{3}>$ or $K \subseteq<\overline{3}>$.

Now we recall some more properties according to Proposition 18 of Raggi et al. [7] which showed the relation between a fully prime submodule and the factor module. If a submodule $N$ is fully prime, then the factor module $M / N$ is also fully prime. But we need some property for the converse, as we can see below.

Proposition 2.4. Let $N$ be a proper fully-invariant submodule of $M$.

(i) If $N$ is fully prime in $M$, then $M / N$ is a fully prime module.

(ii) If $M$ is self-projective and $M / N$ is fully prime, then $N$ is a fully prime submodule in $M$. 
Based on Proposition 2.4, if a module $M$ is self-projective, then a submodule $P$ in $M$ is fully prime if and only if $M / P$ is fully prime. Let $L$ and $U$ be $R$-modules and recall the following definition:

$$
\operatorname{Rej}(L, U)=\bigcap\left\{\operatorname{Ker} f \mid f \in \operatorname{Hom}_{R}(L, U)\right\}
$$

An $R$-module $L$ is called $U$-cogenerated if $\operatorname{Rej}(L, U)=0$. For detailed explanation of reject and cogenerator, the readers are suggested to refer to Wisbauer's book [10]. Moreover, we modify the result in 3.1 of [8] for a more general case.

Proposition 2.5. Let $P$ be a fully invariant submodule of a self-projective module $M$. The following statements are equivalent:

a. $P$ is a fully prime submodule;

b. $\operatorname{Rej}(M / P, K / P)=0$ for any non-zero fully invariant submodules $K / P$ of $M / P$;

c. $K *_{M} L \nsubseteq P$ for any non-zero fully invariant submodules $K / P$ and $L / P$ of $M / P$;

d. $\operatorname{Rej}(-, M / P)=\operatorname{Rej}(-, K / P)$ for any non-zero fully invariant submodules $K / P$ of $M / P$.

According to Proposition 2.5, if $P$ is fully prime, then any $M / P$-cogenerated module is also $K / P$-cogenerated and vice versa.

Consider $R$ as a left $R$-module and let $I, J$ be ideals of $R$. Then $I *_{R} J=I J$. Since every ideal of $R$ is a fully invariant $R$-submodule, we get the following special case:

Proposition 2.6. [8] The following statements are equivalent for a two-sided ideal $I$ :

a. $R / I$ is a prime ring.

b. $I$ is a fully prime submodule in $R$.

c. I is a prime ideal.

A module $M$ satisfies the $(* f i)$ condition if for any non-zero fully invariant submodule $K$ of $M, \operatorname{Ann}_{R}(M / K) \not \subset \operatorname{Ann}_{R}(M)$. A module $M$ is called $f$ retractable if for any non-zero fully invariant submodule $K$ of $M, \operatorname{Hom}_{R}(M, K)=$ $\operatorname{Ann}_{S}(M / K) \neq 0$.

In general prime modules in the sense of Dauns [2] need not to be fully prime. Furthermore, in any self-projective module, if its submodule is prime, then it is not necessary fully prime. For the following relationship we generalize Proposition 3.4 of [8] as follows.

Proposition 2.7. For a self-projective $R$-module $M$ with $(* f i)$ and for any fully invariant submodules $K$ of $M$, the following statements are equivalent:

a. $K$ is prime and $M / K$ fi-retractable.

b. $K$ is fully prime. 
Notice that for any $\operatorname{ring} R, \operatorname{End}_{R}(R) \simeq R$ and as a left $R$-module, $R$ satisfies (*fi) and is fi-retractable. If $M=R$, Corollary 3.5 and Proposition 3.4 of [8] show that primeness and fully primeness of $R$ coincide.

For any fully invariant submodule $K$ of $M$ we denote

$$
(K: M):=\{f \in S \mid M f \subseteq K\}=\operatorname{Ann}_{S}(M / K) .
$$

Now we recall the definition of prime submodule in the sense of Sanh et al. [5], and we called it N-prime, as follows.

Definition 2.8. Let $K$ be a fully invariant and proper submodule of $M$. The submodule $K$ is called an $N$-prime submodules in $M$ if for any fully invariant submodules $L$ of $M$ and for every ideal $I$ of $S$, if $I(L) \subset K$, then $I(M) \subseteq K$ or $L \subset K$.

We refer to Theorem 1.2 of Sahn et. al. [5] to give a characterization of an N-prime submodule and consider that the prime notion in the sense of Sanh (N-prime) and endo-prime notion in the sense of Haghany-Vedadi [3] coinside. As an immediate consequence we extend Corollary 1.6 of [3] as follows.

Proposition 2.9. Suppose ${ }_{R} M$ is fi-retractable and satisfies the $(* f i)$ condition. Then ${ }_{R} M$ is prime if and only if ${ }_{R} M$ is $N$-prime.

As a consequence of Proposition 2.7 and Proposition 2.9 we have the following property.

Lemma 2.10. Suppose ${ }_{R} M$ is fi-retractable, satisfies the $(* f i)$ condition and is self-projective. If $M$ is fully prime, then $M$ is $N$-prime.

Lemma 2.10 gives a sufficient condition of a fully prime module to be N-prime. Now we refer to Theorem 1.2 of Sahn et. al. [5] to prove the following property.

Proposition 2.11. Let $M$ be an R-module, fi-retractable, satisfies the $(* f i)$ condition and self-projective. Let $S=\operatorname{End}_{R}(M)$. If $P$ is a fully prime submodule, then $(P: M)$ is a prime ideal in $S$.

Proof. Take any $f \in S$, an ideal $T$ of $S$ where $f T \subseteq(P: M)$. Then $(M) f T \subseteq P$. Moreover we have $(M) f S T \subseteq(M) f T \subseteq P$. According to Theorem 1.2 [5], since $P$ is fully prime, $P$ is $N$-prime, i.e. $(M) f S \subseteq P$ or $M T \subseteq P$. We show that $f S \subseteq(P: M)$ or $T \subseteq(P: M)$. Thus $(P: M)$ is a prime ideal of $S$.

Related to fully prime submodules, we observe now the notion below.

Definition 2.12. Let $X$ be a non empty subset of a module $M$ where $0 \notin X$. $X$ is called a fully $m$-system if for any $x, y \in X,\langle x\rangle *_{M}<y>\cap X \neq \emptyset$.

As it is already known, there is a similar m-system notion in rings and it is a complement set of a prime ideal (see for example Lam [4]). In modules, we prove that the fully m-system is also a complement set of a fully prime submodule.

Proposition 2.13. Let $P$ be a fully invariant submodule of $M$. $P$ is fully prime if and only if $M \backslash P$ is a fully m-system. 
Proof. $(\Rightarrow)$. Denote $X=M \backslash P$. Take any $x, y \in X$, then $x, y \notin P$. By Proposition 2.2 (b), $\left\langle x>*_{M}<y>\nsubseteq P\right.$, hence $\left\langle x>*_{M}<y>\cap X \neq \emptyset\right.$. Hence $X=M \backslash P$ is a fully m-system.

$(\Leftarrow)$. Take any $x, y \notin P$, then $x, y \in M \backslash P$ and $\left\langle x>*_{M}<y>\nsubseteq \nsubseteq P\right.$. Hence $P$ is fully prime.

Next we show that any maximal fully invariant submodule is also a fully prime submodule.

Proposition 2.14. Let $X$ be a fully $m$-system in $M$ and $P$ be a maximal fully invariant submodule in $M$ where $P \cap X=\emptyset$. Then $P$ is a fully prime submodule in $M$.

Proof. Suppose $x \notin P$ and $y \notin P$, but $\left\langle x>*_{M}<y>\subseteq P\right.$. Then there exist $x_{1}, x_{2} \in X$ such that $x_{1} \in P+\langle x\rangle$ and $x_{2} \in P+\langle y\rangle$. Consider that

$$
\begin{aligned}
<x_{1}>*_{M}<x_{2}> & \subseteq(P+<x>) *_{M}(P+<y>) \\
& \subseteq P+\left(<x>*_{M}<y>\right) \subseteq P .
\end{aligned}
$$

This is a contradiction with the fact that $X$ is a fully $\mathrm{m}$-system.

Moreover, we also show that any fully prime submodule contains a minimal fully prime submodule.

Definition 2.15. A fully invariant submodule $N$ in $M$ is called minimal fully prime if $N$ is minimal in the set of all fully prime submodules in $M$.

Proposition 2.16. Let $P$ be a fully prime submodule in $M$. Then $P$ contains a minimal fully prime submodule.

Proof. Let $P$ be a fully prime submodule in $M$. We form the following set

$$
\mathfrak{J}=\{U \mid U \text { fully prime submodule in } M \text { where } U \subseteq P\} .
$$

It is clear that $\mathfrak{J} \neq \emptyset$, since $P \in \mathfrak{J}$. By Zorn's Lemma, $\mathfrak{J}$ has a minimal element or equivalently, every nonempty chain in $\mathfrak{J}$ has a lower bound in $\mathfrak{J}$.

Consider a nonempty chain $\mathfrak{G} \subseteq \mathfrak{J}$. We construct a set $Q=\bigcap_{K \in \mathfrak{G}} K$. It is clear that $Q$ is a fully invariant submodule in $M$ and $Q \subseteq P$. We want to show that $Q$ is a fully prime submodule in $M$. Take any two fully invariant submodules $X$ and $Y$ in $M$ where $X *_{M} Y \subseteq Q$ but $Y \nsubseteq Q$. We prove that $X \subseteq Q$. Take any $y \in Y \backslash Q$. Then there exists $K^{\prime} \in \mathfrak{G}$ such that $y \notin K^{\prime}$. Since $K^{\prime}$ is a fully prime submodule in $M$, from $X *_{M} Y \subseteq Q \subseteq K^{\prime}$ implies $X \subseteq K^{\prime}$.

Then take any $L \in \mathfrak{G}$. Since $\mathfrak{G}$ is a chain in $\mathfrak{J}, K^{\prime} \subseteq L$ or $L \subseteq K^{\prime}$. If $K^{\prime} \subseteq L$, then $X \subseteq K^{\prime} \subseteq L$. If $L \subseteq K^{\prime}$, then $y \notin L$. From $X *_{M} Y \subseteq Q \subseteq L$ implies $X \subseteq L$. Thus $X \subseteq L$ for all $L \in \mathfrak{G}$. Then $X \subseteq Q$, and we prove that $Q$ is a fully prime submodule in $M$.

Since $Q \subseteq P, Q \in \mathfrak{J}$ and is a lower bound of $\mathfrak{G}$. It is proved that any nonempty chain in $\mathfrak{J}$ has a lower bound in $\mathfrak{J}$. Based on Zorn's Lemma there exists a fully prime submodule $P^{*} \in \mathfrak{J}$ which is minimal in $\mathfrak{J}$. Thus the fully prime submodule $P$ contains the minimal fully prime submodule $P^{*}$. 
Let $N$ and $K$ be fully invariant submodules of $M$ where $K \subseteq N$. We consider then the sets $\operatorname{Hom}_{R}(M, K)$ and $\operatorname{Hom}_{R}(N, K)$. For any $f \in \operatorname{Hom}_{R}(M, K)$, it induces a homomorphism $\tilde{f}=\left.f\right|_{N} \in \operatorname{Hom}_{R}(N, K)$. It is understood that there exists an injective function from $\operatorname{Hom}_{R}(M, K)$ to $\operatorname{Hom}_{R}(N, K)$ which maps any $f \in$ $\operatorname{Hom}_{R}(M, K)$ to $\tilde{f}=\left.f\right|_{N} \in \operatorname{Hom}_{R}(N, K)$. Hence, $\operatorname{Hom}_{R}(M, K) \subseteq \operatorname{Hom}_{R}(N, K)$.

Proposition 2.17. Let $N$ and $P$ be a fully invariant submodules of $M$. If $P$ is fully prime, then $N \cap P$ is a fully prime submodule in $N$.

Proof. Take any two fully invariant submodules $K$ and $L$ in $N$, where $L *_{N} K \subseteq$ $N \cap P$. Then $L *_{N} K \subseteq P$ and $L \operatorname{Hom}_{R}(N, K) \subseteq P$. Since $\operatorname{Hom}_{R}(M, K) \subseteq$ $\operatorname{Hom}_{R}(N, K), L \operatorname{Hom}_{R}(M, K) \subseteq L \operatorname{Hom}_{R}(N, K)$. Moreover $L *_{M} K \subseteq L *_{N} K$ and hence $L *_{M} K \subseteq P$. Then $L \subseteq P$ or $K \subseteq P$ because $P$ is fully prime. But $K \subseteq N$ and $L \subseteq N$, so we have $K \subseteq N \cap P$ or $L \subseteq N \cap P$ as well.

We recall Lemma 17 of Raggi et al. [7] below.

Lemma 2.18. Let $M$ and $N$ be $R$-module and $f \in \operatorname{Hom}_{R}(M, N)$ an epimorphism.

(i) If $\operatorname{Ker} f$ is a fully invariant submodule in $M$ and $L$ is a fully invariant submodule in $N$, then $(L) f^{-1}$ is a fully invariant submodule in $M$.

(ii) If $M$ is a quasi-projective module and $U$ is a fully invariant submodule in $M$, then $(U) f$ is a fully invariant submodule in $N$.

As a consequence, we have the following property.

Corollary 2.19. Let $M$ be R-module and $K, U$ submodules of $M$ where $K \subset U$ and $K$ fully invariant in $M$. If $U / K$ is a proper fully invariant submodule in $M / K$, then $U$ is a proper fully invariant submodule in $M$.

Proposition 2.20. Let $M$ be a self-projective module and $A, P$ fully invariant submodules in $M$ where $A \subset P$. The submodule $P$ is a fully prime submodule in $M$ if and only if $P / A$ is a fully prime submodule in $M / A$.

Proof. Let $\bar{S}=\operatorname{End}_{R}(M / A)$.

$(\Rightarrow)$. We want to prove that $P / A$ is a fully prime submodule in $M / A$. Take any fully invariant submodule $K / A$ and $L / A$ in $M / A$ such that:

$$
K / A *_{M / A} L / A=K / A \operatorname{Hom}_{R}(M / A, L / A)=\sum_{f \in \operatorname{Hom}_{R}(M / A, L / A)}(K / A) f \subseteq P / A .
$$

Since $K / A$ and $L / A$ is fully invariant submodules in $M / A$, according to Corollary 2.19 we get $K$ and $L$ are fully invariant in $M$.

Now take any $f \in \operatorname{Hom}_{R}(M / A, L / A) \subseteq \bar{S}$. Since $M$ is a self-projective module, there exists a homomorphism $g \in \operatorname{Hom}_{R}(M, L)$ such that $\pi f=g \pi$, where $\pi$ is the natural epimorphism $\pi: M \rightarrow M / A$. Then

$$
(K / A) f=K(\nu f)=K(g \nu)=(K g) \nu=(K g+A) / A \subseteq P / A .
$$

Thus we have $K g \subseteq P$.

Hence for any $f \in \operatorname{Hom}_{R}(M / A, L / A)$ there exists $g \in S$ such that $K g \subseteq P$. 
Moreover, $\sum_{g \in S} K g=K S \subseteq P$. Since $K_{\operatorname{Hom}_{R}}(M, L)=K *_{M} L \subseteq K S \subseteq P$, it implies $K \subseteq P$ or $L \subseteq P$. We obtain $K / A \subseteq P / A$ or $L / A \subseteq P / A$, i.e. $P / A$ is a fully prime submodule in $M / A$.

$(\Leftarrow)$. Let $P / A$ be a fully prime submodule in $M / A$. Take any fully invariant submodule $K$ and $L$ in $M$ where:

$$
K *_{M} L \subseteq K \operatorname{Hom}_{R}(M, L) \subseteq \sum_{f \in \operatorname{Hom}_{R}(M, L)} K f \subseteq P .
$$

Based on Lemma 2.18 (ii), we obtain that $(K+A) / A$ and $(L+A) / A$ are fully invariant submodules in $M / A$. Now take any $f \in \operatorname{Hom}_{R}(M, L)$ or equivalently $f \in S$. Since $A$ is a fully invariant submodule in $M$, we can construct the following function:

$$
\begin{aligned}
h: M / A & \rightarrow M / A \\
m+A & \mapsto(m+A) h=m f+A, \text { for any } m+A \in M / A .
\end{aligned}
$$

Take any $m+A, n+A \in M A$ and $r \in R$, then:

$$
\begin{aligned}
((m+A)+(n+A)) h & =((m+n)+A) h \\
& =(m+n) f+A \\
& =(m f+n f)+A \\
& =(m f+A)+(n f+A) \\
& =((m+A) h)+((n+A) h) .
\end{aligned}
$$

and

$$
(r(m+A)) h=(r m+A) h=(r m) f+A=r(m f+A)=r(m+A) h .
$$

It is proved that $h$ is a homomorphism, so we get $h \in \bar{S}$. Now take any $m \in M$. For any natural epimorphism $\nu: M \rightarrow M A$ it yields

$$
m(\nu h)=(m \nu) h=(m+A) h=m f+A=(m f) \nu=m(f \nu) .
$$

It implies $\nu h=f \nu$.

Furthermore, $\sum_{f \in \operatorname{Hom}_{R}(M, L)} K f \subseteq P$ implies $K f \subseteq P$. As a consequence, $K(f \nu)=$ $(K f) \nu \subseteq P \nu=P / A$. We use the fact that $\nu h=f \nu$ to obtain $K(\nu h) \subseteq P / A$. Then $((K+\bar{A}) A) h \subseteq P / A$ and $\sum_{h \in \bar{S}}((K+A) A) h=((K+A) A) \bar{S} \subseteq P / A$.

Since

$$
(K+A) / A \operatorname{Hom}_{R}(M / A,(L+A) / A) \subseteq((K+A) / A) \bar{S} \subseteq P / A,
$$

we have $((K+A) / A) *_{M / A}((L+A) / A) \subseteq P / A$. Since $P / A$ is a fully prime submodule in $M / A$, we get $(K+A) / A \subseteq P / A$ or $(K+A) / A \subseteq P / A$. Hence $K \subseteq P$ or $L \subseteq P$. 


\section{Fully Prime Radicals}

Now we collect all fully prime submodules in $M$ in the spectrum of $M$ below. We denote the fully prime radical of $L$ as $\operatorname{Rad}_{M}^{f p}(L)$, where $L$ is a fully invariant submodule of $M$.

$$
\begin{aligned}
\operatorname{Spec}^{f p}(M) & :=\{K \supsetneqq M \mid K \text { is fully prime submodule in } \mathrm{M}\} \\
\nu^{f p}(L) & :=\left\{K \in \operatorname{Spec}^{f p}(M) \mid L \subseteq K\right\} \\
\operatorname{Rad}_{M}^{f p}(L) & :=\bigcap_{K \in \nu^{f p}(L)} K
\end{aligned}
$$

In case a fully prime submodule which contains $L$ does not exist, we define $\operatorname{Rad}_{M}^{f p}(L)=M$. It is easy to understand that $\nu^{f p}(0)=\operatorname{Spec}^{f p}(M)$ and $\nu^{f p}(M)=\emptyset$.

For a special case, we have $\operatorname{Rad}_{M}^{f p}(0)=\bigcap_{K \in \nu^{f p}(0)} K$. If $M$ contains fully prime submodules, then

$$
\operatorname{Rad}_{M}^{f p}(0)=\bigcap_{K \in \operatorname{Spec}_{M}^{f p}(M)} K .
$$

However, if there are no fully prime submodules in $M$, then $\operatorname{Rad}_{M}^{f p}(0)=M$. We call the $\operatorname{Rad}_{M}^{f p}(0)$ the fully prime radical of $M$. of $L$.

The following proposition gives us a characterization of the fully prime radical

Proposition 3.1. Let $L$ be a fully invariant submodule of $M$. Denote

$$
\mathcal{R}=\{x \in M \mid \forall \text { fully } \mathrm{m}-\text { system } X, x \in X \Rightarrow X \cap L \neq \emptyset\} .
$$

Then we have $\operatorname{Rad}_{M}^{f p}(L)=M$ or $\operatorname{Rad}_{M}^{f p}(L)=\mathcal{R}$.

Proof. First, we show that $\operatorname{Rad}_{M}^{f p}(L) \subseteq \mathcal{R}$. Take any $y \notin \mathcal{R}$ and prove that $y \notin$ $\operatorname{Rad}_{M}^{f p}(L)$. Since $y \notin \mathcal{R}$, there is a fully m-system $X^{\prime}$ such that $y \in X^{\prime}$ but $X^{\prime} \cap L=\emptyset$. Consider now

$$
\mathcal{M}=\left\{N \subseteq M \mid N \text { is fully invariant } L \subseteq N, N \cap X^{\prime}=\emptyset\right\},
$$

and a chain $\mathcal{C}$ in $\mathcal{M}$.

The set $\bigcup_{N \in \mathcal{M}} N$ is an upper bound of $\mathcal{C}$. Hence by Zorn's Lemma, there is a maximal element in $\mathcal{M}$, say $N^{\prime}$. It is clear that $N^{\prime}$ is a maximal fully invariant submodule of $M$ and $N^{\prime} \cap X^{\prime}=\emptyset$. According to Proposition 2.14, $N^{\prime}$ is fully prime and $y \notin N^{\prime}$. It implies $y \notin \operatorname{Rad}_{M}^{f p}(L)$.

Conversely, we show that $\mathcal{R} \subseteq \operatorname{Rad}_{M}^{f p}(L)$. Take any $x \in \mathcal{R}$ and $N \in \nu^{f p}(L)$. Then $M \backslash N$ is a fully m-system. Suppose $x \in M \backslash N$ or equivalently $x \notin N$. It implies $(M \backslash N) \cap L \neq \emptyset$. But $L \subseteq N$ and $N \cap L \neq \emptyset$, a contradiction. Thus $x \notin M \backslash N$ or equivalently $x \in N$.

Especially, if $L$ is the zero submodule of $M$, then we have the following property. 
Proposition 3.2. Let $M$ be an R-module. Then $\operatorname{Rad}_{M}^{f p}(0)=M$ or $\operatorname{Rad}_{M}^{f p}(0)=$ $\cap P^{\prime}$ where the $P^{\prime}$ s are minimal fully prime submodules of $M$.

Proof. If $\operatorname{Rad}_{M}^{f p}(0) \neq M$, then $M$ contains a fully prime submodule and $\operatorname{Spec}^{f p}(M) \neq$ $\emptyset$. According to Proposition 2.16, we know that any fully prime submodule in $M$ contains a minimal fully prime submodule. Then for any $P \in \operatorname{Spec}^{f p}(M)$, there exists a minimal fully prime submodule $P^{\prime} \in \operatorname{Spec}^{f p}(M)$ and $P^{\prime} \subseteq P$.

Now we form the following set:

$$
\mathfrak{J}=\left\{P^{\prime} \in \operatorname{Spec}^{f p}(M) \mid P^{\prime} \text { is a minimal fully prime submodule }\right\} .
$$

We want to show $\operatorname{Rad}_{M}^{f p}(0)=\bigcap_{P^{\prime} \in \mathfrak{J}} P^{\prime}$. Since $\mathfrak{J} \subseteq \operatorname{Spec}^{f p}(M), \operatorname{Rad}_{M}^{f p}(0) \subseteq \bigcap_{P^{\prime} \in \mathfrak{J}} P^{\prime}$. Conversely, take any $a \notin \operatorname{Rad}_{M}^{f p}(0)$, then there exists a minimal fully prime submodule $\bar{P} \in \operatorname{Spec}^{f p}(M)$ such that $a \notin \bar{P}$. Consequently $a \notin \bigcap_{P^{\prime} \in \mathfrak{J}} P^{\prime}$, and we prove

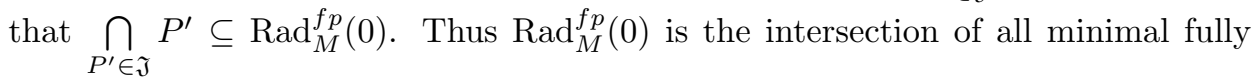
prime submodules in $M$.

Proposition 3.3. Let $\left\{L_{\lambda}\right\}$ be a family of fully invariant submodules in $M$. Then

$$
\bigcap_{\lambda \in \Lambda} \nu^{f p}\left(L_{\lambda}\right)=\nu^{f p}\left(\sum_{\lambda \in \Lambda} L_{\lambda}\right)
$$

Proof. Take any $X \in \bigcap_{\lambda \in \Lambda} \nu^{f p}\left(L_{\lambda}\right)$ and then $X \in \nu^{f p}\left(L_{\lambda}\right)$ for all $\lambda \in \Lambda$. It implies that $X$ is a fully prime submodule which contains $L_{\lambda}$ for all $\lambda \in \Lambda$. Hence $X$ also contains the sum $\sum_{\lambda \in \Lambda} L_{\lambda}$ and $X \in \nu^{f p}\left(\sum_{\lambda \in \Lambda} L_{\lambda}\right)$.

Conversely, now take $Y \in \nu^{f p}\left(\sum_{\lambda \in \Lambda} L_{\lambda}\right)$. It means $Y$ is a fully prime submodule and $\sum_{\lambda \in \Lambda} L_{\lambda} \subseteq Y$. Then for all $\lambda \in \Lambda$ we obtain $L \in \nu^{f p}\left(L_{\lambda}\right)$ since $L_{\lambda} \subseteq Y$. Hence $Y \in \nu^{f p}\left(\sum_{\lambda \in \Lambda} L_{\lambda}\right)$.

Proposition 3.4. Let $M$ be a left $R$-modul, $N$ and $L$ fully invariant submodules of $M$. Then $\nu^{f p}(N) \cup \nu^{f p}(L) \subseteq \nu^{f p}(N \cap L)$.

Proof. Let $P \in \nu^{f p}(N) \cup \nu^{f p}(L)$. We have $P \in \nu^{f p}(N)$ or $P \in \nu^{f p}(L)$. As a consequence we obtain $N \subseteq P$ or $L \subseteq P$. Hence, $N \cap L \subseteq N \subseteq P$ or $N \cap L \subseteq L \subseteq P$. Thus we conclude that $N \cap L \subseteq P$, or in other words $\bar{P} \in \nu^{\bar{f} p}(N \cap L)$.

Let $I$ be an ideal in $S$. Then we denote

$$
\operatorname{Rad}_{S}^{f p}(I)=\cap\{J \mid I \subseteq J, J \text { is a prime ideal in } S\}
$$

Proposition 3.5. If $L$ is a fully invariant submodule of $M$, then $M \operatorname{Rad}_{S}^{f p}((L$ : $M) \subseteq \subseteq \operatorname{Rad}_{M}^{f p}(L)$.

Proof. We have the following two cases. If $\operatorname{Rad}_{M}^{f_{p}}(L)=M$, since $\operatorname{Rad}_{S}^{f p}((L: M)) \subseteq$ $S$, then $\operatorname{Rad}_{S}^{f p}((L: M)) \subseteq M=\operatorname{Rad}_{M}^{f p}(L)$.

If $\operatorname{Rad}_{M}^{f_{p}}(L) \neq M$, then $\nu^{f p}(L) \neq \emptyset$. Moreover $\operatorname{Rad}_{M}^{f_{p}}(L)=\bigcap_{X \in \nu^{f p}(L)} X$. Take any 
$X \in \nu^{f p}(L)$, it means $X$ a fully prime submodule in $M$ where $L \subseteq X$. According to Proposition $2.11(X: M)$ is a prime ideal in $S$ where $(L: M) \subseteq(X: M)$. Then $\operatorname{Rad}_{S}^{f p}((L: M)) \subseteq(X: M)$ and moreover

$$
\operatorname{Rad}_{S}^{f p}((L: M)) \subseteq M(X: M) \subseteq X .
$$

We conclude then

$$
M \operatorname{Rad}_{S}^{f p}((L: M)) \subseteq \bigcap_{X \in \nu^{f p}(L)} X=\operatorname{Rad}_{M}^{f_{p}}(L) .
$$

Proposition 3.6. Let $N$ be a fully invariant submodule of $M$. Then, $\operatorname{Rad}_{N}^{f_{p}}(0) \subseteq$ $\operatorname{Rad}_{M}^{f p}(0)$.

Proof. Take any fully prime submodule $P \in \operatorname{Spec}^{f p}(M)$. If $N \subseteq P$, then the intersection of all fully prime submodule of $N$ is contained in $P$. Moreover we have $\operatorname{Rad}_{N}^{f p}(0) \subseteq P$. Now if $N \nsubseteq P$, then according to Proposition 2.17 we conclude that $N \cap P$ is a fully prime submodule in $N$. Hence $\operatorname{Rad}_{N}^{f p}(0) \subseteq N \cap P \subseteq P$. It is proved that $\operatorname{Rad}_{N}^{f p}(0) \subseteq \operatorname{Rad}_{M}^{f p}(0)$.

Proposition 3.7. Let $\left\{N_{i}\right\}$ be the family of fully invariant submodules of $M$. If $M=\bigoplus_{i \in I} N_{i}$, a direct sum of fully invariant submodules $N_{i}$ s in $M$, then

$$
\bigoplus_{i \in I} \operatorname{Rad}_{N_{i}}^{f p}(0) \subseteq \operatorname{Rad}_{M}^{f p}(0)
$$

Proof. It is obvious.

It is easy to prove the following property.

Proposition 3.8. Let $M$ be an $R$-module and $P_{1}, P_{2}$ any fully prime submodules in $M$. If $P_{1} / \operatorname{Rad}_{M}^{f p}(0)$ and $P_{2} / \operatorname{Rad}_{M}^{f p}(0)$ are submodules in $M / \operatorname{Rad}_{M}^{f p}(0)$, then:

$$
P_{1} / \operatorname{Rad}_{M}^{f p}(0) \cap P_{2} / \operatorname{Rad}_{M}^{f p}(0)=\left(P_{1} \cap P_{2}\right) / \operatorname{Rad}_{M}^{f p}(0) .
$$

Proposition 3.9. If $M$ is a self-projective module, then: $\operatorname{Rad}_{M}^{f p}\left(M / \operatorname{Rad}_{M}^{f p}(M)\right)=$ $\overline{0}$.

Proof. If $M$ does not contain a fully prime submodule, then based on Proposition 2.20 we get that $M / \operatorname{Rad}_{M}^{f p}(0)$ also does not contain any fully prime submodule. So $\operatorname{Rad}_{M}^{f p}=M$ and:

$$
\operatorname{Rad}_{M}^{f p}\left(M / \operatorname{Rad}_{M}^{f p}(0)\right)=\operatorname{Rad}_{M}^{f p}(M / M)=\operatorname{Rad}_{M}^{f p}(\overline{0})=\overline{0} .
$$

Now assume $M$ has a fully prime submodule. According to Proposition 2.20 we get that $M / \operatorname{Rad}_{M}^{f p}(0)$ also contains a fully prime submodule. Then we have :

$$
\operatorname{Rad}_{M}^{f p}\left(M / \operatorname{Rad}_{M}^{f p}(0)\right)=\bigcap_{\bar{P} \in \operatorname{Spec}^{f p}\left(M / \operatorname{Rad}_{M}^{f p}(0)\right)} \bar{P} .
$$


We apply Proposition 3.8 to get the following:

$$
\begin{aligned}
\operatorname{Rad}_{M}^{f p}\left(M / \operatorname{Rad}_{M}^{f p}(0)\right) & =\bigcap_{\bar{P} \in \operatorname{Spec}_{p p}\left(M / \operatorname{Rad}_{M}^{f p}(0)\right)} \bar{P} \\
& =\left(\bigcap_{P \in \operatorname{Spec}^{f p}(M)} P\right) \operatorname{Rad}_{M}^{f p}(0) \\
& =\operatorname{Rad}_{M}^{f p}(0) / \operatorname{Rad}_{M}^{f p}(0) \\
& =\overline{0}
\end{aligned}
$$

\section{Concluding Remarks}

Further work on the properties of fully prime radicals of submodules can be carried out. For example, we can define a fully multiplication module and then investigate the properties of fully prime radicals on the fully multiplication module. Moreover, we can bring this work to define fully prime localizations and then investigate the fully prime localization on fully multiplication modules.

Acknowledgement. We thank the referees for their time and comments.

\section{REFERENCES}

[1] Azizi,A.: Radical formula and prime submodules, Journal of Algebra, 307 (2007), 454 - 460.

[2] Dauns,J.: Prime Modules, Journal für die reine und angewandte Mathematik, 298 (1978), $156-181$.

[3] Haghany,A., Vedadi,M.R.: Endoprime Modules, Acta Mathematica Hungaria, 106 (1-2) (2005), 89-99.

[4] Lam,T.Y.: A First Course in Noncommutative Rings, Springer-Verlag, New York, 1991.

[5] Sanh,N.V., Nguyen,A.V., Ahmed,K.F.U., Asawasamrit,S., Thao,L.P.: Primeness in Module Category, Asian-European Journal of Mathematics, 3(1) (2010), 145-154.

[6] Sanh,N.V., Thao, L.P., Al-Mayahi,N.F.A., Shum,K.P.: Zariski Topology of Prime Spectrum of a Module, Proceedings of the International Conference on Algebra 2010 ed. Wanida Hemakul et. al. (World Scientific), (2012), 461-477.

[7] Raggi,F., Ríos,J., Rincón,H., Fernández-Alonso,R., Signoret,C.: Prime and Irreducible Preradicals, J. Algebra Appl., 4(4) (2005), 451 - 466.

[8] Wijayanti,I.E., Wisbauer,R.: Coprime Modules and Comodules, Communications in Algebra, 37 (4) (2009), 1308 - 1333

[9] Wijayanti,I.E.: On Left Residuals of Submodules in Fully Multiplication Modules, JP Journal of Algebra, Number Theory and Applications, 36 (1) (2015), 17 - 28.

[10] Wisbauer,R.: Foundations of Module and Ring Theory A handbook for Study and Research, Gordon and Breach Science Publishers, Philadelphia, 1991.

[11] Wisbauer,R.: Modules and algebras : bimodule structure and group actions on algebras, Addison Wesley Longman Ltd., Essex, 1996. 